\title{
Vulnerabilidad sísmica estructural de instituciones educativas públicas, aplicando el método de benedetti-petrini
}

\section{Structural seismic vulnerability of public educational institutions, applying the benedetti-petrini method}

\author{
Cristian Moreto Tuesta ${ }^{1}$, María Jose Mechato Jiménez ${ }^{2}$, Guillermo Arturo Díaz Jáuregui ${ }^{3}$
}

\section{RESUMEN}

La presente investigación tuvo como objetivo evaluar la vulnerabilidad sísmica estructural de tres instituciones educativas públicas del centro histórico de la ciudad de Chachapoyas en la región Amazonas Perú. Se evaluaron tres instituciones educativas: Virgen Asunta, Institución Educativa Isabel Lynch de Rubio y la Institución Educativa Seminario Jesús María, aplicando el método de Benedetti y Petrini. Se encontró que la vulnerabilidad sísmica de la Institución Educativa Virgen Asunta, es BAJA, excepto los módulos C y D que presentan una vulnerabilidad MEDIA A BAJA. La vulnerabilidad sísmica de la Institución Educativa Isabel Lynch de Rubio, es BAJA, excepto los módulos D y E que presentan una vulnerabilidad MEDIAA BAJA. La vulnerabilidad sísmica de la Institución Educativa Seminario Jesús María, es BAJA. Finalmente se determinó que el Peligro Sísmico de las tres Instituciones Educativas Públicas del Centro Histórico de Chachapoyas en evaluación es MEDIO y la estimación del Riesgo Sísmico de las 03 Instituciones Educativas es MEDIO.

Palabras clave: Vulnerabilidad sísmica, peligro sísmico, riesgo

\begin{abstract}
The objective of this research was to evaluate the structural seismic vulnerability of three public educational institutions in the historic center of the city of Chachapoyas in the Amazon region of Peru. Three educational institutions were evaluated: Virgen Asunta, Isabel Lynch de Rubio Educational Institution and the Jesús María Seminary Educational Institution, applying the method of Benedetti and Petrini. It was found that the seismic vulnerability of the Virgen Asunta Educational Institution is LOW, except for modules C and D that present a MEDIUM TO LOW vulnerability. The seismic vulnerability of the Isabel Lynch de Rubio Educational Institution is LOW, except for modules D and E that present a MEDIUM TO LOW vulnerability. The seismic vulnerability of the Jesús María Seminary Educational Institution is LOW. Finally, it was determined that the Seismic Hazard of the three Public Educational Institutions of the Historic Center of Chachapoyas under evaluation is MEDIUM and the estimate of the Seismic Risk of the 03 Educational Institutions is MEDIUM.
\end{abstract}

Keywords: Seismic vulnerability, seismic hazard, risk

\footnotetext{
${ }^{1}$ Bachiller en Ingeniería Civil de la Universidad Nacional Toribio Rodríguez de Mendoza de Amazonas. Correo: electrónico:cristianmoretot@hotmail.com.

${ }^{2}$ Bachiller en Ingeniería Civil de la Universidad Nacional Toribio Rodríguez de Mendoza de Amazonas. Correo electrónico: majomj161718@hotmail.com.

${ }^{3} \mathrm{Mg}$. Gestión Pública, docente de la escuela profesional de ingeniería civil de la UNTRM. Correo electrónico: arturo.diaz@untrm.edu.pe
} 


\section{INTRODUCCIÓN}

El Perú se encuentra en la zona de más alta actividad sísmica del mundo, y ciudades como Chachapoyas han sido afectadas por sismos, generando perjuicios, por vulnerabilidad del suelo, tipo de estructura y la práctica de autoconstrucción, por ende, un Estudio de Vulnerabilidad Sísmica Estructural se convierte en una necesidad.

Las experiencias de sismos en todo el mundo, han manifestado la sobresaliente vulnerabilidad de las edificaciones esenciales, ya que la mayoría de ellas han fallado estructural o funcionalmente, incapacitadas de asistir a la población antes y a posteriori de un evento catastrófico. Por este motivo, se han implementado metodologías que permiten catalogar y cuantificar la vulnerabilidad de las edificaciones (Camacho, 2011).

Los desastres dejan en evidencia que los programas de Riesgo Sísmico no se han aplicado para esquivar o mitigar estas pérdidas, exigido a la falta de recursos económicos designados a la investigación y tecnología para reforzar estudios de su prevención y mitigación. La universalidad de las pérdidas generadas por terremotos, han sido causadas por una deficiente disposición sísmica de las estructuras, llegando a colapsar parcial o totalmente (Caballero, 2007).

Es así que, Mosqueira (2012), en su indagación de los edificios de la facultad de ingeniería de la Universidad Nacional de Cajamarca, determinó el riesgo sísmico con el fin de estimar el comportamiento sísmico de las edificaciones y de esta manera prevenir los daños que se ocasionaría. Sus resultados indicaron que las edificaciones tenían un riesgo sísmico alto y frente a un sismo, se generarían problemas de columna corta y desplome de la pared por la ausencia de elementos de confinamiento.

En el mismo contexto, Abanto \& Cardenas (2015), en su incumbencia sobre la vulnerabilidad sísmica para el centro histórico de Trujillo, tomaron como muestra dos instituciones educativas públicas. Utilizaron el método de Benedetti y Petrini (Italia), concluyendo que en general, las estructuras de ambas instituciones educativas tienen vulnerabilidad media baja, así como una infraestructura de albañilería confinada construidas sin previo criterio técnico de edificaciones sismorresistentes.

Con el mismo fin, Oc (2017) en su investigación sobre el riesgo sísmico de las viviendas del barrio Santa Isabel en Chachapoyas, evaluó el peligro sísmico y la vulnerabilidad de las viviendas, para luego estimar el riesgo sísmico. Para el peligro sísmico utilizó el método probabilístico que consiste en elaborar leyes de atenuación en base a registros históricos, para lo cual usó el software Crisis 2007. Para evaluar la vulnerabilidad utilizó el método italiano propuesto por Benedetti y Petrini. Finalmente, para el cálculo del riesgo sísmico trabajó con el método descriptivo que se apoya en el uso de la Matriz de Peligro y vulnerabilidad, la cual interrelaciona el peligro y la vulnerabilidad. Obteniendo como resultados que el $34.4 \%$ de las viviendas tienen riesgo sísmico intermedio, $62.3 \%$ riesgo sísmico alto y $3.3 \%$ riesgo sísmico muy alto.

\section{MATERIAL Y MÉTODO}

La presente investigación estudió la vulnerabilidad sísmica estructural de tres instituciones educativas públicas del Centro Histórico de Chachapoyas aplicando el método de Benedetti y Petrini, adaptándolo al Reglamento Nacional de Edificaciones, las cuales fueron: Institución Educativa Virgen Asunta, Institución Educativa Seminario Jesús María e Institución Educativa Isabel Lynch de Rubio.

Las instituciones educativas estudiadas están ubicadas dentro del centro histórico de Chachapoyas.

\section{Figura 1}

Ubicación de las Instituciones Educativas.

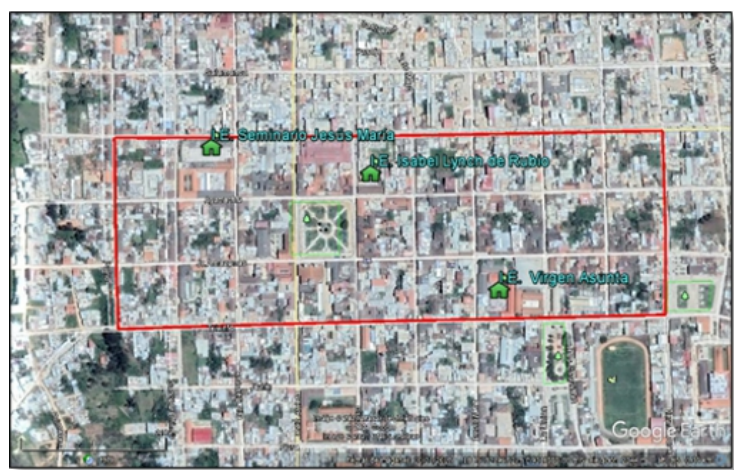

\section{MÉTODO}

El procedimiento se apoyó en calificar los edificios por medio de la inspección visual de sus propiedades físicas, con ciertos cálculos primordiales, identificando los daños más relevantes en las estructuras generados por un sismo, obteniendo así una calificación de la calidad del diseño y la construcción sismorresistente de los edificios por medio de un coeficiente nombrado Índice de Vulnerabilidad (Iv) (Abanto \& Cardenas, 2015).

Se obtuvo por medio de una suma ponderada de los valores numéricos que manifiestan la "calidad sísmica" de todos los parámetros, a cada uno se le clasifica en una de las clases. La calificación "A" es óptima con un valor numérico $\mathrm{Ki}=0$, mientras que la 
"D" es la más desfavorable con un valor numérico $\mathrm{Ki}=45$, como se observa en la Tabla 1 (Marin, 2012).

\section{Tabla 1}

Escala numérica del índice de vulnerabilidad "Iv" de los edificios de mampostería no reforzada (Benedetti y Petrini, 1984).

\begin{tabular}{|c|c|c|c|c|c|c|}
\hline $\mathbf{i}$ & Parámetro & $\mathbf{K i}^{*} \mathbf{A}$ & $\mathbf{K i}^{*} \mathbf{B}$ & $\mathrm{Ki}^{\star} \mathbf{C}$ & $\mathbf{K i}^{*} \mathbf{D}$ & $\mathbf{w}$ \\
\hline 1 & $\begin{array}{l}\text { Organización del } \\
\text { sistema resistente }\end{array}$ & 0 & 5 & 20 & 45 & 1,00 \\
\hline 2 & $\begin{array}{l}\text { Calidad del } \\
\text { sistema resistente }\end{array}$ & 0 & 5 & 25 & 45 & 0,25 \\
\hline 3 & $\begin{array}{l}\text { Resistencia } \\
\text { convencional } \\
\text { Posición del }\end{array}$ & 0 & 5 & 25 & 45 & 1,50 \\
\hline 4 & $\begin{array}{l}\text { edificio y } \\
\text { cimentación }\end{array}$ & 0 & 5 & 25 & 45 & 0,75 \\
\hline 5 & $\begin{array}{l}\text { Diafragmas } \\
\text { horizontales }\end{array}$ & 0 & 5 & 15 & 45 & 1,00 \\
\hline 6 & $\begin{array}{l}\text { Configuración en } \\
\text { planta }\end{array}$ & 0 & 5 & 25 & 45 & 0,50 \\
\hline 7 & $\begin{array}{l}\text { Configuración en } \\
\text { elevación }\end{array}$ & 0 & 5 & 25 & 45 & 1,00 \\
\hline 8 & $\begin{array}{l}\text { Distancia entre } \\
\text { columnas }\end{array}$ & 0 & 5 & 25 & 45 & 0,25 \\
\hline 9 & Tipo de cubierta & 0 & 15 & 25 & 45 & 1,00 \\
\hline 10 & $\begin{array}{l}\text { Elementos no } \\
\text { estructurales }\end{array}$ & 0 & 0 & 25 & 45 & 0,25 \\
\hline 11 & $\begin{array}{l}\text { Estado de } \\
\text { conservación }\end{array}$ & 0 & 5 & 25 & 45 & 1,00 \\
\hline
\end{tabular}

\section{Fuente: Marín 2012}

Con los 11 parámetros cuantificados en cada una de las clases (A, B, C o D), se determinó el Índice de Vulnerabilidad, mediante una sumatoria del valor de cada parámetro multiplicado por un peso, mostrada en la siguiente ecuación:

$$
I v=\sum_{i=1}^{11}(K i * W i)
$$

\section{Fórmula (1)}

De acuerdo con la Tabla 2, el I.V. de la edificación está entre 0 y 382,5, significando que mientras el puntaje sea más alto, más vulnerable es la edificación. Los valores de Ki y Wi, son plenamente subjetivos y se lograron a partir de la vivencia de sus creadores, cada parámetro es perjudicado por un peso "Wi", que está entre 0,25 y 1,50 (Marín, 2012).
Tabla 2

Rango de Valores del Índice de Vulnerabilidad.

\begin{tabular}{ccc}
\hline SISMICIDAD & $\begin{array}{c}\text { PELIGRO } \\
\text { SÍSMICO }\end{array}$ & \multicolumn{1}{c}{ RANGO } \\
\hline \multirow{2}{*}{ ALTA } & Bajo & 1,80 \\
& Medio & De 2,00 a 2,40 \\
& Alto & De 2,60 a 3,00 \\
\hline \multirow{2}{*}{ MEDIA } & Bajo & De 1,40 a 1,60 \\
& Medio & De 1,80 a 2,40 \\
& Alto & 2,60 \\
\hline \multirow{2}{*}{ BAJO } & Bajo & De 1,00 a 1,60 \\
& Medio & De 1,80 a 2,00 \\
& Alto & 2,20 \\
\hline
\end{tabular}

Fuente: (Abanto \& Cardenas, 2015).

Metodología para determinar el peligro sísmico

Para determinar el peligro sísmico. Tucto (2018) en su investigación consideró 3 factores importantes: "la Sismicidad, el Tipo de Suelo y la Topografía del área donde se desarrolla dicha investigación". En este caso se consideró las Instituciones Educativas en evaluación, calculándose mediante la siguiente ecuación: Peligro Sísmico $=(0,40 *$ Sismicidad $)+$ $(0,40 *$ Suelo $)+(0,20 *$ Topografía $)$

Para obtener la designación del Peligro Símico, se reemplazaron los valores numéricos de las variables de la Tabla 3 en la ecuación.

Tabla 3

Cuantificación del Peligro Sísmico.

\begin{tabular}{|c|c|c|c|c|c|}
\hline \multicolumn{2}{|c|}{$\begin{array}{c}\text { SISMICIDA } \\
\text { D (40\%) }\end{array}$} & \multicolumn{2}{|c|}{$\begin{array}{c}\text { SUELO } \\
(40 \%)\end{array}$} & \multicolumn{2}{|c|}{$\begin{array}{c}\text { TOPOGRAFÍA } \\
(20 \%)\end{array}$} \\
\hline Baja & 1 & Rígido & 1 & Plano & 1 \\
\hline Media & 2 & $\begin{array}{c}\text { Interme } \\
\text { dio }\end{array}$ & 2 & Medio & 2 \\
\hline Alta & 3 & Flexible & 3 & $\begin{array}{c}\text { Pronunci } \\
\text { adas }\end{array}$ & 3 \\
\hline
\end{tabular}

Fuente: (Mosqueira \& Tarque, 2005).

Los valores calculados se ubicaron en los rangos del Peligro Sísmico mostrado en la Tabla 4, para así conocer el Grado de Peligro Sísmico de las Instituciones Educativas. VULNERABILIDADRANGOS (Iv) PORCENTAJE \% A = BAJA 0 - 95,63 $0-25 \% \mathrm{~B}=$ MEDIA A BAJA $95,63-191,3025 \%-50 \% \mathrm{C}$ 
$=$ MEDIAAALTA $191,30-286,3050 \%-75 \% \mathrm{D}=$ ALTA $286,30-382,5075 \%-100 \%$.

\section{Tabla 4}

Calificación del Peligro Sísmico.

\begin{tabular}{cccc}
\hline $\begin{array}{c}\text { VULNERABILI } \\
\text { DAD }\end{array}$ & RANGOS (Iv) & $\begin{array}{c}\text { PORCENTA } \\
\text { JE } \%\end{array}$ \\
\hline $\mathrm{A}=$ & BAJA & $0-95,63$ & $0-25 \%$ \\
$\mathrm{~B}=$ & $\begin{array}{l}\text { MEDIA } \\
\text { A BAJA }\end{array}$ & $95,63-191,30$ & $25 \%-50 \%$ \\
$\mathrm{C}=$ & $\begin{array}{l}\text { MEDIA A } \\
\text { ALTA }\end{array}$ & $191,30-286,30$ & $50 \%-75 \%$ \\
$\mathrm{D}=$ & ALTA & $286,30-382,50$ & $75 \%-100 \%$ \\
& &
\end{tabular}

Fuente: (Mosqueira \& Tarque, 2005).

\section{Metodología para estimar el riesgo sísmico}

Para designar el Nivel de Riesgo Símico, se reemplazaron los valores de Vulnerabilidad y Peligro Sísmico en la Tabla 5.

\section{Tabla 5}

Nivel de Riesgo Sísmico.

\begin{tabular}{ccccc}
\hline \multicolumn{4}{c}{ RIESGO SÍSMICO } \\
\hline & Vulnerabilidad & Baja & Media & Alta \\
\hline Peligro & & & & \\
\hline & Bajo & BAJO & MEDIO & MEDIO \\
& Medio & MEDIO & MEDIO & ALTO \\
Alto & MEDIO & ALTO & ALTO \\
\hline
\end{tabular}

Fuente: "Recomendaciones Técnicas para Mejorar la Seguridad Sísmica de Viviendas de Albañilería Confinada de la Costa Peruana". (Mosqueira \& Tarque, 2005).

Riesgo Sísmico Alto; supone que, al ocurrir un sismo severo, la edificación sufrirá daños importantes con riesgo de que colapse al no tener adecuado comportamiento sísmico, además de ubicarse sobre suelo blando (Mosqueira \& Tarque, 2005).

Riesgo Sísmico Medio, supone que, las edificaciones tienes un aceptable comportamiento sísmico, y al ocurrir un sismo, los pórticos y muros de la edificación podrían sufrir daños menores y algunos tabiques podrían colapsar parcial o totalmente. (Mosqueira \& Tarque, 2005).

Riesgo Sísmico Bajo, supone que, el estado de conservación de la edificación es regular a buena, cimentada sobre un suelo rígido sin pendiente y que, ante la ocurrencia de un sismo, tiene un adecuado comportamiento sísmico y solo podría sufrir el colapso parcial o total de sus tabiques (Mosqueira \& Tarque, 2005).

\section{RESULTADOS}

Tabla 6

Vulnerabilidad (Iv) de las Instituciones Educativas.

\begin{tabular}{cccc}
\hline \multicolumn{5}{c}{ COLEG VIRGEN ASUNTA } \\
\hline MODULO & Iv & Iv $\%$ & VULNE RABILIDAD \\
\hline A & 93.75 & $24.51 \%$ & BAJA \\
B & 82.50 & $21.57 \%$ & BAJA \\
C & 105.00 & $27.45 \%$ & MEDIA A BAJA \\
D & 105.00 & $27.45 \%$ & MEDIA A BAJA \\
E & 88.75 & $23.20 \%$ & BAJA \\
F & 52.50 & $13.73 \%$ & BAJA \\
G & 71.25 & $18.63 \%$ & BAJA \\
H & 73.75 & $19.28 \%$ & BAJA \\
I & 83.75 & $21.90 \%$ & BAJA \\
\hline COLEGIO ISABEL LYNCH DE RUBIO \\
\hline A & 57.50 & $15.03 \%$ & BAJA \\
B & 52.50 & $13.73 \%$ & BAJA \\
C & 78.75 & $20.59 \%$ & BAJA \\
D & 147.50 & $38.56 \%$ & MEDIA A BAJA \\
E & 138.75 & $36.27 \%$ & MEDIAA BAJA \\
F & 87.50 & $22.88 \%$ & BAJA \\
G & 52.50 & $13.73 \%$ & BAJA \\
H & 68.75 & $17.97 \%$ & BAJA \\
I & 67.50 & $17.65 \%$ & BAJA \\
J & 77.50 & $20.26 \%$ & BAJA \\
\hline COLEGIO SEMINARIO JESU S MARIA \\
\hline A & 72.50 & $18.95 \%$ & BAJA \\
B & 72.50 & $18.95 \%$ & BAJA \\
C & 72.50 & $18.95 \%$ & BAJA \\
D & 72.50 & $18.95 \%$ & BAJA \\
E & 63.75 & $16.67 \%$ & BAJA \\
F & 32.50 & $8.50 \%$ & BAJA \\
G & 52.50 & $13.73 \%$ & BAJA \\
H & 67.50 & $17.65 \%$ & BAJA \\
\hline \multicolumn{5}{c}{} & & \\
\hline
\end{tabular}


Tabla 7

Peligro Sísmico de las Instituciones Educativas.

\begin{tabular}{|c|c|c|c|c|c|c|c|c|}
\hline COLEGIO & \multicolumn{2}{|c|}{ SISMICDAD } & \multicolumn{2}{|l|}{ SUELO } & \multicolumn{2}{|c|}{ TOPOGRAFIA } & \multicolumn{2}{|c|}{ PELIGRO SÍSMICO } \\
\hline \multicolumn{9}{|c|}{ IE VIRGEN ASUNTA } \\
\hline MÓDULO A & MEDIA & 2 & INTERMEDIO & 2 & PLANA & 1 & 1.8 & MEDIO \\
\hline MÓDULO B & MEDIA & 2 & INTERMEDIO & 2 & PLANA & 1 & 1.8 & MEDIO \\
\hline \multicolumn{9}{|l|}{ PABELLÓN 2} \\
\hline $\begin{array}{l}\text { MÓDULO C } \\
\text { PABELLÓN } 3\end{array}$ & MEDIA & 2 & INTERMEDIO & 2 & PLANA & 1 & 1,8 & MEDIO \\
\hline MÓDULO D & MEDIA & 2 & INTERMEDIO & 2 & PLANA & 1 & 1.8 & MEDIO \\
\hline \multicolumn{9}{|l|}{ PABELLÓN 4} \\
\hline $\begin{array}{l}\text { MÓDULO E } \\
\text { PABELLÓN } 5\end{array}$ & MEDIA & 2 & INTERMEDIO & 2 & PLANA & 1 & 1.8 & MEDIO \\
\hline \multicolumn{9}{|l|}{ PABELLÓN 6} \\
\hline MÓDULO G & MEDIA & 2 & INTERMEDIO & 2 & PLANA & 1 & 1,8 & MEDIO \\
\hline MÓDULO H & MEDIA & 2 & INTERMEDIO & 2 & PLANA & 1 & 1.8 & MEDIO \\
\hline \multicolumn{9}{|l|}{ PABELLÓN 7} \\
\hline MÓDULO I & MEDIA & 2 & INTERMEDIO & 2 & PLANA & 1 & 1.8 & MEDIO \\
\hline \multicolumn{9}{|c|}{ IE ISABEL LYNCH DE RUBIO } \\
\hline \multicolumn{9}{|l|}{ PABELLÓN 1} \\
\hline MÓDULO A & MEDIA & 2 & INTERMEDIO & 2 & PLANA & 1 & 1.8 & MEDIO \\
\hline \multicolumn{9}{|l|}{ PABELLÓN 2} \\
\hline $\begin{array}{l}\text { MÓDULO B } \\
\text { PABELLÓN } 3\end{array}$ & \multicolumn{7}{|c|}{ PABELLÓN 3} & MEDIO \\
\hline MÓDULO C & MEDIA & 2 & INTERMEDIO & 2 & PLANA & 1 & 1.8 & MEDIO \\
\hline \multicolumn{9}{|l|}{ PABELLÓN 4} \\
\hline $\begin{array}{l}\text { MÓDULO D } \\
\text { PABELLÓN } 5\end{array}$ & \multicolumn{7}{|c|}{ PABELLÓN 5} & MEDIO \\
\hline MÓDULO E & MEDIA & 2 & INTERMEDIO & 2 & PLANA & 1 & 1.8 & MEDIO \\
\hline \multicolumn{9}{|l|}{ PABELLÓN 6} \\
\hline \multicolumn{8}{|l|}{ PABELLÓN 7} & MEDIO \\
\hline MÓDULO G & MEDIA & 2 & INTERMEDIO & 2 & PLANA & 1 & 1.8 & MEDIO \\
\hline \multicolumn{9}{|l|}{ PABELLÓN 8} \\
\hline MÓDULO H & MEDIA & 2 & INTERMEDIO & 2 & PLANA & 1 & 1,8 & MEDIO \\
\hline MÓDULO I & MEDIA & 2 & INTERMEDIO & 2 & PLANA & 1 & 1.8 & MEDIO \\
\hline MÓDULO J & MEDIA & 2 & INTERMEDIO & 2 & PLANA & 1 & 1.8 & MEDIO \\
\hline \multicolumn{9}{|l|}{ I.E SEMINARIO JE: } \\
\hline \multicolumn{9}{|l|}{ PABELLÓN 1} \\
\hline MÓDULO A & MEDIA & 2 & INTERMEDIO & 2 & PLANA & 1 & 1,8 & MEDIO \\
\hline MÓDULO B & MEDIA & 2 & INTERMEDIO & 2 & PLANA & 1 & 1,8 & MEDIO \\
\hline MÓDULO C & MEDIA & 2 & INTERMEDIO & 2 & PLANA & 1 & 1,8 & MEDIO \\
\hline \multicolumn{9}{|l|}{ PABELLÓN 2} \\
\hline MÓDULO D & MEDIA & 2 & INTERMEDIO & 2 & PLANA & 1 & 1,8 & MEDIO \\
\hline MÓDULO E & MEDIA & 2 & INTERMEDIO & 2 & PLANA & 1 & 1,8 & MEDIO \\
\hline PABELLÓN 3 & & & & & & & & \\
\hline MÓDULO F & MEDIA & 2 & INTERMEDIO & 2 & PLANA & 1 & 1,8 & MEDIO \\
\hline MÓDULO G & MEDIA & 2 & INTERMEDIO & 2 & PLANA & 1 & 1,8 & MEDIO \\
\hline MÓDULO H & MEDIA & 2 & INTERMEDIO & 2 & PLANA & 1 & 1,8 & MEDIO \\
\hline
\end{tabular}


Tabla 8

Nivel de Riesgo Sísmico de las Instituciones Educativas.

\begin{tabular}{|c|c|c|c|}
\hline COLEGIO & $\begin{array}{c}\text { NIVEL DE PE LIGRO } \\
\text { SÍSMICO }\end{array}$ & $\begin{array}{c}\text { NIVE L DE } \\
\text { VULNERABILIDAD }\end{array}$ & $\begin{array}{l}\text { NIVEL DE } \\
\text { RIESGO }\end{array}$ \\
\hline \multicolumn{4}{|c|}{ I.E VIRGEN ASUNTA } \\
\hline \multicolumn{4}{|c|}{ PABELLÓN 1} \\
\hline MÓDULO A & MEDIO & BAJA & MEDIO \\
\hline MÓDULO B & MEDIO & BAJA & MEDIO \\
\hline \multicolumn{4}{|l|}{ PABELLÓN 2} \\
\hline MÓDULO C & MEDIO & MEDIA A BAJA & MEDIO \\
\hline \multicolumn{4}{|l|}{ PABELLÓN 3} \\
\hline MÓDULO D & MEDIO & MEDIA A BAJA & MEDIO \\
\hline \multicolumn{4}{|l|}{ PABELLÓN 4} \\
\hline MÓDULO E & MEDIO & BAJA & MEDIO \\
\hline \multicolumn{4}{|l|}{ PABELLÓN 5} \\
\hline MÓDULO F & MEDIO & BAJA & MEDIO \\
\hline \multicolumn{4}{|l|}{ PABELLÓN 6} \\
\hline MÓDULO G & MEDIO & BAJA & MEDIO \\
\hline MÓDULO H & MEDIO & BAJA & MEDIO \\
\hline \multicolumn{4}{|l|}{ PABELLÓN 7} \\
\hline MÓDULO I & MEDIO & BAJA & MEDIO \\
\hline \multicolumn{4}{|c|}{ I.E ISABEL LYNCH DE RUBIO } \\
\hline \multicolumn{4}{|l|}{ PABELLÓN 1} \\
\hline MÓDULO A & MEDIO & BAJA & MEDIO \\
\hline \multicolumn{4}{|l|}{ PABELLÓN 2} \\
\hline MÓDULO B & MEDIO & BAJA & MEDIO \\
\hline \multicolumn{4}{|l|}{ PABELLÓN 3} \\
\hline MÓDULO C & MEDIO & BAJA & MEDIO \\
\hline \multicolumn{4}{|l|}{ PABELLÓN 4} \\
\hline MÓDULO D & MEDIO & MEDIA A BAJA & MEDIO \\
\hline \multicolumn{4}{|l|}{ PABELLÓN 5} \\
\hline MÓDULO E & MEDIO & MEDIA A BAJA & MEDIO \\
\hline \multicolumn{4}{|l|}{ PABELLÓN 6} \\
\hline MÓDULO F & MEDIO & BAJA & MEDIO \\
\hline \multicolumn{4}{|l|}{ PABELLÓN 7} \\
\hline MÓDULO G & MEDIO & BAJA & MEDIO \\
\hline \multicolumn{4}{|l|}{ PABELLÓN 8} \\
\hline MÓDULO H & MEDIO & BAJA & MEDIO \\
\hline MÓDULO I & MEDIO & BAJA & MEDIO \\
\hline MÓDULO J & MEDIO & BAJA & MEDIO \\
\hline \multicolumn{4}{|c|}{ I.E SEMINARIO JESÚS MARÍA } \\
\hline \multicolumn{4}{|l|}{ PABELLÓN 1} \\
\hline MÓDULO A & MEDIO & BAJA & MEDIO \\
\hline MÓDULO B & MEDIO & BAJA & MEDIO \\
\hline MÓDULO C & MEDIO & BAJA & MEDIO \\
\hline \multicolumn{4}{|l|}{ PABELLÓN 2} \\
\hline MÓDULO D & MEDIO & BAJA & MEDIO \\
\hline MÓDULO E & MEDIO & BAJA & MEDIO \\
\hline \multicolumn{4}{|l|}{ PABELLÓN 3} \\
\hline MÓDULO F & MEDIO & BAJA & MEDIO \\
\hline MÓDULO G & MEDIO & BAJA & MEDIO \\
\hline MÓDULO H & MEDIO & BAJA & MEDIO \\
\hline
\end{tabular}




\section{DISCUSIÓN}

Según el método (Benedetti \& Petrini, 1984), utilizado para la evaluación de la vulnerabilidad sísmica de las instituciones educativas del Centro Histórico de Chachapoyas, se pudo resaltar que en los parámetros que más vulnerabilidad presentan, son: configuración en planta, tipos de cubierta, elementos no estructurales y estado de conservación, además presentan deficiencias en el asentado de ladrillos, juntas inadecuadas de separación entre los muros y los elementos estructurales, datos que probablemente se debe a que algunos de los módulos de estos edificios se construyeron con la Primera Norma Peruana de Diseño Sismorresistente oficializada en 1977 y otros con la Norma Técnica E030 Diseño Sismorresistente de 1997 (Muñoz, 2020); vale poner énfasis en que en ambas normas no se consideraban los desplazamientos laterales de los edificios o estructuras, permitiendo estructuras con elementos alargados e incluso hasta esbeltos. Así mismo se pudo observar que las tres instituciones educativas presentan problemas de humedad y eflorescencia en sus losas aligeradas y muros.

Se observó en su mayoría, que los módulos presentaron un inadecuado confinamiento de los muros a los elementos estructurales, omitiendo la construcción de viguetas y columnetas, a consecuencia de estos aspectos constructivos se genera la llamada columna corta en sus estructuras. Los muros reforzados con la técnica propuesta en la presente investigación ante esta problemática, podrían comportarse de manera eficiente ante un evento sísmico como lo sostuvieron San Bartolomé et al., (2007) en su estudio experimental. En vista de que la presente investigación fue descriptiva y no se consideró simulaciones con efectos sísmicos, se recomienda que en investigaciones futuras similares sean abordados los mencionados efectos.

En la ciudad de Chachapoyas, no se realizaron estudios de vulnerabilidad sísmica, peligro sísmico y riesgo sísmico para Instituciones Educativas en general, solo se realizaron estudios para viviendas.

Como es el caso de Oc, (2017), quien en su investigación sobre la estimación del riesgo sísmico de las viviendas autoconstruidas en el barrio Santa Isabel de Chachapoyas, sostuvo que, de las viviendas evaluadas, un 3.3\% tienen una VULNERABILIDAD MUY ALTA, 59\% tienen una VULNERABILIDAD ALTA y $34.5 \%$ tienen una VULNERABILIDAD MEDIAABAJA. Respecto al peligro sísmico, obtuvo un PELIGRO SÍSMICO ALTO y la mayoría de las viviendas evaluadas presentaron un RIESGO SÍSMICO ALTO. Comparando con los resultados obtenidos en este proyecto de investigación, deducimos que estos difieren, ya que la vulnerabilidad sísmica de las tres Instituciones Educativas es BAJA, a excepción de 2 módulos de la institución educativa Isabel Lynch de
Rubio y 2 módulos de la institución educativa Virgen Asunta, donde los resultados se asemejan ya que es MEDIAABAJA; y concerniente al estudio de peligro sísmico y riesgo sísmico de las Instituciones Educativas son de estimación MEDIA, por lo tanto, concluimos que son resultados totalmente opuestos, esto se debe a que en el caso de Oc, (2017), muchas de las viviendas fueron construidas con adobe y sin la intervención de mano de obra calificada, omitiendo las recomendaciones del RNE.

Con el mismo fin, Mosqueira M. A. (2012), en su investigación sobre la determinación del riesgo sísmico de las edificaciones de la facultad de ingeniería de la Universidad Nacional de Cajamarca, obtuvo que esta edificación presenta un RIESGO SÍSMICO ALTO, PELIGRO SÍSMICO ALTO y VULNERABILIDAD SÍSIMICA ALTA, contrastando con los resultados de nuestra tesis, concluimos que estos difieren ya que obtuvimos un RIESGO SÍSMICO MEDIO, PELIGRO SÍSMICO MEDIO, VULNERABILIDAD BAJA Y VULNERABILIDAD MEDIA A BAJA. Estas diferencias se deben a que las edificaciones evaluadas se encuentran en una zona de alta sismicidad.

En su estudio de investigación Hidalgo \& Silvestre (2019), hicieron uso del método de Benedetti Petrini para una institución educativa ubicada en el distrito y provincia de Barranca, llegando a obtener como resultados una VULNERABILIDAD BAJA hasta VULNERABILIDAD MEDIA ALTA, resultados que se asemejan y difieren a nuestra investigación correspondientemente, ya que se obtuvo un resultado de VULNERABILIDAD BAJA y VULNERABILIDAD MEDIA A BAJA. Estos autores recomiendan un reforzamiento estructural, y además consideran que el problema principal de las estructuras es su configuración en planta y su falta de rigidez; el problema de configuración en planta es típico también en nuestra investigación, debido a que la propagación de las ondas sísmicas depende del suelo y básicamente de la altura, provocando aceleraciones diferentes con esfuerzos longitudinales de tensión y de compresión. De ahí que cuando más largo sea el edificio, mayor será la probabilidad de estos esfuerzos y mayor será su efecto.

En el mismo sentido Abanto \& Cardenas (2015), en su investigación, determinó la Vulnerabilidad Sísmica de las edificaciones de las Instituciones Educativas Públicas del Centro Histórico de Trujillo, con el fin de fomentar acciones de mitigación ante un evento sísmico, haciendo uso también del método de Benedetti y Pettrini (Italia). Los resultados que obtuvieron fueron estructuras con VULNERABILIDAD MEDIA BAJA, sosteniendo que las instituciones educativas evaluadas son infraestructuras de albañilería confinada que posiblemente fueron construidas sin criterio técnico de edificación sismorresistente. Respecto a estos resultados, podemos afirmar que los resultados obtenidos en la 
presente investigación, sobre la Vulnerabilidad Sísmica mediante el mismo método de las instituciones educativas en muestra investigación, también fueron de VULNERABILIDAD BAJA y MEDIA A BAJA, ya que no tuvieron asesoría profesional durante su etapa de ejecución y debido a su antigüedad no siguieron las normas técnicas ni recomendaciones del R.N.E. Norma Técnica E-070 Albañilería.

Para la construcción de instituciones educativas futuras tomando en cuenta aspectos que afectan el nivel de vulnerabilidad sísmica, se debe regir al Reglamento Nacional de edificaciones, poniendo énfasis en la Norma Técnica E-030 Diseño Sismorresistente y la Norma Técnica E-070 Albañilería.

Respecto a las fisuras y grietas encontradas en los muros de albañilería de los módulos, se recomienda hacer uso de las técnicas de reparación detalladas en la sección anterior.

Para los problemas de columna corta encontrados en la mayoría de los módulos, se recomienda como alternativas de solución el ensanche a un lado de las columnas con espesor igual al del alféizar, detalladas en la sección anterior.

\section{CONCLUSIONES}

La vulnerabilidad sísmica de la Institución Educativa Virgen Asunta, es BAJA, excepto los módulos C y D que presentan una vulnerabilidad MEDIAABAJA.

La vulnerabilidad sísmica de la Institución Educativa Isabel Lynch de Rubio, es BAJA, excepto los módulos $\mathrm{D}$ y $\mathrm{E}$ que presentan una vulnerabilidad MEDIAABAJA.

La vulnerabilidad sísmica de la Institución Educativa Seminario Jesús María, es BAJA.

Se determinó que el Peligro Sísmico de las tres Instituciones Educativas Públicas del Centro Histórico de Chachapoyas en evaluación es MEDIO.

La estimación del Riesgo Sísmico de las 03 Instituciones Educativas es MEDIO. Ya que este resultado depende de la vulnerabilidad y el peligro sísmico calculado anteriormente.

Los módulos tienen aceptable comportamiento sísmico y ante un evento sísmico los pórticos y muros de los módulos podrían sufrir daños menores.

\section{REFERENCIAS BIBLIOGRÁFICAS}

Abanto, S., \& Cardenas, D. J. (2015). Determinación de la vulnerabilidad sísmica aplicando el método de benedetti - petrini en las instituciones educativas del centro histórico de Trujillo, provincia de Trujillo, región La Libertad (Tesis de Pregrado). Universidad Privada Antenor Orrego, Trujillo.

Benedetti, D., \& Petrini, V. (1984). "Sulla vulnerabilitá sismica di edifici in muratura $i$ propostedi un metodo di vautazione". L'industria delle Construzioni.

Caballero, A. R. (2007). Determinación de la vulneravilidad sísmica por medio del método del índice de vulnerabilidad en las estructuras ubicadas en el centro histórico de la ciudad de Sincelejo, utilizando la tecnología del sistema de información geográfica (Tesis de Maestría). Universidad del Norte, Sincelejo.

Camacho, A. V. (2011). Vulnerabilidad sísmica estructural de los edificios principales de la facultad de ingeniería civil de la universidad nacional de ingeniería (Tesis de Pregrado). Universidad Nacional de Ingeniería, Lima.

Hidalgo, E. J., \& Silvestre, R. J. (2019). Evaluación de la Vulnerabilidad Sísmica de la Institución Educativa $N^{o} 20475$ - Los Pelones, del Distrito y Provincia de Barranca del Departamento de Lima. (tesis de Pregrado). Universidad Nacional José Faustino Sanchez Carrión, Huacho.

Llanos, L. F., \& Vidal, L. M. (2003). Evaluación de la vulnerabilidad sísmica de escuelas públicas de Cali: una propuesta metodológica (Tesis de Pregrado). Universidad del Valle, Santiago de Cali.

Marin, F. (2012). Evaluación del riesgo sísmico del centro histórico de la ciudad de Huánuco (Tesis de Maestría). Universidad Nacional de Ingeniería, Lima.

Mosqueira, M. A. (2012). Riesgo sísmico en las edificaciones de la facultad de ingeniería, Universidad Nacional de Cajamarca, Perú. Escuela de Postgrado. Universidad Nacional de Trujillo, La Libertad.

Mosqueira, M. Á., \& Tarque, S. N. (2005). Recomendaciones Técnicas para Mejorar la Seguridad (Tesis de Maestría). Pontífica Universidad Católica del Perú, Lima.

Muñoz, A. (2020). Comentarios a la Norma Peruana E.030 Diseño Sismoresistente. SENCICO, 
Lima.

Oc, J. (2017). Estimación del riesgo sísmico de las viviendas autoconstruidas en el barrio Santa Isabel, Chachapoyas, Amazonas, 2016 (Tesis de Pregrado). Universidad Nacional Toribio Rodriguez de Mendoza de Amazonas, Chachapoyas.

RNE. (2018). Norma Técnica E-030 Diseño Sismorresistente. Lima, Perú.

RNE. (2018). Norma Técnica E-070 Albañilería. Lima, Perú.

San Bartolomé, A., Rivera, C., Durán, M., Muñoz, A., \& Quiun, D. (2007). Estudio experimental de una técnica de reforzamiento para edificaciones existentes con problemas de columna corta Proyecto SENCICO - PUCP. Pontifica Universidad Catolica del Perú, Lima.

Tucto, J. D. (2018). Evaluación del Riesgo Sísmico utilizando el Indice de Vulnerabilidad de Benedetti - Petrini en las viviendas de adobe existentes en la zona urbana del distrito de LLacanora, Cajamarca (Tesis de Pregrado). Universidad Nacional de Cajamarca, Cajamarca. 